\title{
Establishing Restricted Expression of Caveolin-1 in HIV Infected Cells and Inhibition of Virus Replication
}

\author{
Yung-Tsun Lo, Peter E. Nadeau, Shanshan Lin and Ayalew Mergia* \\ Department of Infectious Disease and Pathology, University of Florida, Gainesville, FL 32611, USA
}

\begin{abstract}
Background: Caveolin-1 (Cav-1) is the major protein of the caveolae and plays a role in multiple cellular functions and implicated to have anti-HIV activity. Regulated expression of Cav-1 is important for safe and effective use in order to exploit Cav-1 for HIV therapeutic applications.

Methods: A series of Cav-1 and GFP expression vectors were constructed under the control of the HIV LTR for conditional expression or CMV promoter and the expression of Cav-1 was monitored in the presence or absence of Tat or HIV infection in order to establish the restricted expression of Cav-1 to HIV infected cells.

Results: Cav-1 expression was evident under the control of the HIV LTR in the absence of Tat or HIV infection as demonstrated by immunoblot. Placing two internal ribosomal entry sequences (IRES) and a Rev response element, RRE (5' LTR-IRES-GFP-RRE-IRES-Cav-1 3') resulted in no expression of Cav-1 in the absence of Tat with effective expression in the presence of Tat. Transduction of HIV permissive cells with this construct using a foamy virus vector show that Cav-1 was able to inhibit HIV replication by $82 \%$. Cells that received LTR-IRES-GFP-RRE-IRES-Cav-1 remain healthy in the absence of Tat or HIV infection.

Conclusion: These results taken together reveal the inclusion of two IRES establishes a significant reduction of leak through expression of Cav-1 in the absence of Tat or HIV infection. Such regulated expression will have therapeutic application of Cav-1 for HIV infection as well as broad applications which can be beneficial for other host-targeted interventions as therapeutics.
\end{abstract}

Keywords: Caveolin-1, HIV, LTR, Tat.

\section{INTRODUCTION}

Caveolin-1 (Cav-1) is the major protein of the caveolae organelle, a specialized membrane lipid raft that is highly enriched in cholesterol, phospholipids and sphingolipids [1-7]. Caveolae serves as scaffolding for compartmentalized cellular processes and participates in multiple cellular functions, including endocytosis, cholesterol transport, and signal transduction [7-16]. Cav-1 is abundant in various cell types and is highly expressed in quiescent or terminally differentiated cells including dendritic cells and monocytes/ macrophages [3-7, 17-21]. The caveolin scaffolding domain (CSD), residues 82-101 in Cav-1, is essential for both caveolin oligomerization and the interaction of caveolins with other proteins [22]. The CSD serves as receptor for binding proteins containing the sequence with a motif

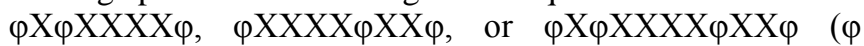
representing any aromatic amino acids and $X$ any other amino acids) [22]. The human immunodeficiency virus (HIV) envelope (Env) gp41 has one such motif

*Address correspondence to this author at the Department of Infectious Disease and Pathology, University of Florida, Gainesville, FL 32611, USA;

Tel: (352)-294-4139; Fax: (352)-392-9704; E-mail:mergiaa@ufl.edu
(WNNMTWMQW) [23, 24] located in the target cells [25, 26]. Through the CSD Cav-1 can interact with ectodomain of gp41 that is required for membrane fusion of with HIV transmembrane gp41 Env [23, 24, 27]. Our group has demonstrated interaction of Cav-1 and HIV Env in the lipid raft (caveolae) of the plasma membrane [28]. Such an interaction at the plasma membrane blocks HIV envelope mediated fusion with target cells and innocent bystander cell killing. Furthermore, the association of Cav-1 with HIV Env reduces virus replication [28]. In addition, Cav-1 can inhibit HIV by reducing nuclear factor карра B (NF- $\mathrm{BB}$ ) mediated transcription and enhancing cholesterol efflux [29-31]. The potential contribution of $\mathrm{Cav}-1$ by suppressing HIV replication can be exploited for the development of a novel therapeutic strategy in treating HIV-1 infected patients. Cav1 , however, has multiple functions including regulating signal transduction, vesicle trafficking and cholesterol metabolism as well as being linked to multiple diseases including cancer [14, 21]. Therefore, overexpressing Cav-1 for therapeutic applications could have an adverse effect due to unregulated expression. To overcome such a problem we established a strategy that significantly restricts Cav-1 expression in HIV infected cells while effectively inhibiting virus replication. 


\section{MATERIALS AND METHODS}

\section{Cell Cultures}

The 293T (human embryonic kidney fibroblasts)cell line was obtained from American Type Culture Collection (Manassas, VA). Glial derived human U87MG-CD4 cells stably transfected with CXCR4 (U87-CD4-CXCR4) and an indicator cell line for tittering HIV (TZM-bl) was kindly provided by the NIH AIDS Reagent Program. U87MG-CD4 derived cells were maintained in Dulbecco's modified Eagle medium (DMEM) containing 15\% FBS, penicillinstreptomycin $(100 \mu \mathrm{g} / \mathrm{mL})$, glutamine $(584 \mu \mathrm{g} / \mathrm{mL})$, puromycin (1 $\mu \mathrm{g} / \mathrm{ml}$; Sigma Chemical), and neomycin (G418; $300 \mu \mathrm{g} / \mathrm{ml}$; Sigma). TZM-bl and 293T cells were grown in DMEM supplemented with $10 \%$ fetal bovine serum (FBS) and penicillin-streptomycin $(100 \mu \mathrm{g} / \mathrm{ml})$.

\section{Construction of Plasmids}

The NIH AIDS Research Program kindly provided pNL4-3 a plasmid construct containing the HIV provirus of NL 4-3. All expression constructs used for the studies are depicted in Fig. (1). The CMV-Cav-1plasmid contains the Cav-1 coding sequence followed by an internal ribosomal entry sequence (IRES) and then green fluorescent protein (GFP) under the control of the human cytomegalovirus (CMV) immediate early gene promoter. CMV-IR is the same as CMV-Cav-1 but lacks the coding sequence of Cav-1. The constructs LTR-IR, LTR-Cav-1, were derived from CMV-IR and CMV-Cav-1, respectively, by replacing the CMV promoter with the HIV NL4-3 LTR. In constructing LTRGFP-IR-Cav-1 we placed the GFP upstream of Cav-1 while placing an IRES in between the two coding sequences with expression under the control of the HIV LTR. An additional IRES was inserted upstream GFP in LTR-GFP-IR-Cav-1 generating the construct LTR-IR-GFP-IR-Cav-1. The plasmid LTR-IR-GFP-RRE-IR-Cav-1 was derived from LTR-IR-GFP-IR-Cav-1 by inserting the HIV Rev response element sequence between GFP and the IRES located upstream Cav-1. RRE was also placed in LTR-IR-GFP upstream IRES and GFP generating plasmid LTR-RRE-IRGFP. To deliver the Cav-1/GFP expression cassettes into HIV target cells foamy virus vectors were constructed using the simian foamy virus type 1 , isolated from rhesus macaque (SFVmac). An SFVmac vector containing GFP (pEGFPD) was used as the backbone to construct foamy virus vectors to deliver into HIV target cells. The construction of pEGFPD and a foamy virus vector expressing a microRNA based siRNA targeting the HIV rev/env (SFV/HL-R) as well as foamy virus vector packaging plasmids pCIgag, pCIpol, and pCIenv expressing gag, polymerase and envelope, respectively were described previously [32]. The GFP expression cassette was removed in pEGFPD and replaced with the expression cassette of GFP and Cav-1 with two IRES and RRE under the control of LTR from LTR-IR-GFPRRE-IR-Cav-1 or a CMV promoter expressing Cav-1 and GFP from CMV-Cav-1 generating SFV/LTR-IR-GFP-RREIR-Cav-1 or SFV/CMV-Cav-1, respectively. A Tat expression plasmid pTatz was described previously [32].

A.

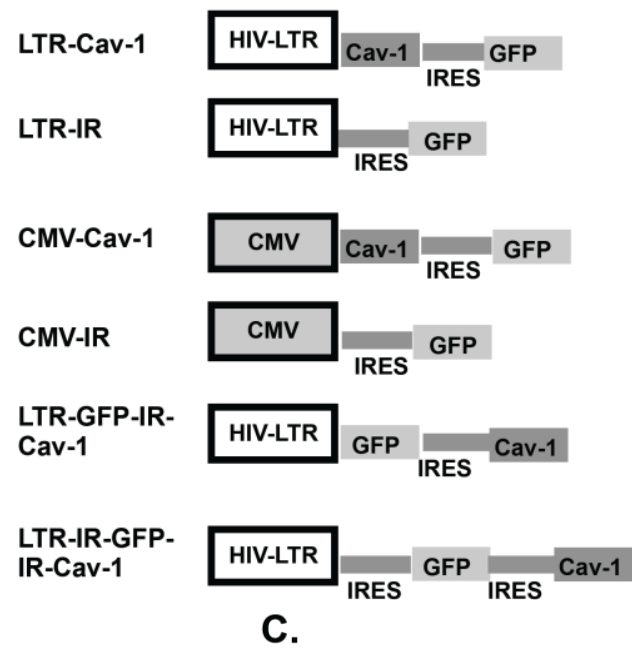

B.
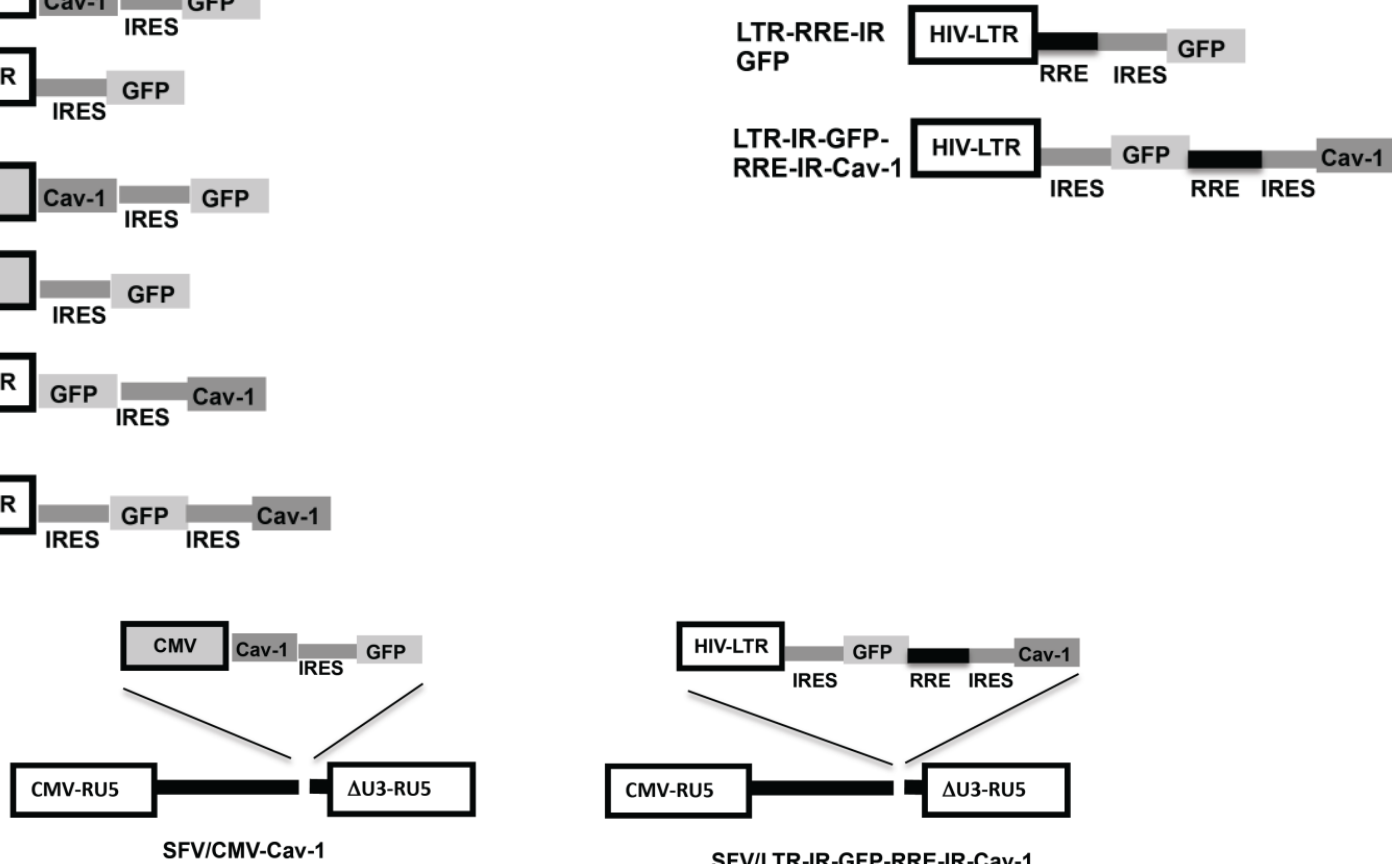

SFVILTR-IR-GFP-RRE-IR-Cav-1

Fig. (1). Schematic representation of Cav-1 and GFP expression constructs. Cav-1 and GFP expression cassettes were generated in the presence of one or two internal ribosomal entry sequence (IRES) (A) plus the Rev response element (RRE) (B). The Cav-1 and GFP expression under the control of the CMV promoter or the LTR for conditional expression were placed in the simian foamy virus isolated from rhesus macaque (SFVmac) vector to deliver into HIV target cells (C). 
DNA Transfection, Cav-1 Expression, and HIV Inhibition Assay

Transfection of each of the plasmid constructs was performed in six well plates by the calcium phosphate method as described previously [33]. Briefly, 293T cells were cultured at a density of $4.5 \times 10^{5}$ in 6 well plates and transfected with $1 \mathrm{ug}$ of each expression construct carrying the Cav-1 and GFP or GFP alone along with 1 ug of plasmid DNA lacking an expression cassette. For induction of LTR promoter mediated expression the Tat expression construct pTatz was co-transfected $(1 \mathrm{ug})$ along with $1 \mathrm{ug}$ of each of the expression cassettes. Twenty-four hours post transfection the culture medium was removed and replaced with fresh media. The transfected cells were kept in culture for 3-4 days while monitoring for GFP expression under a microscope with ultraviolet source. The numbers of fluorescent cells were scored in the presence and absence of Tat. In addition, the levels of expression of Cav-1 and GFP were examined by Western blot and GFP by the intensity of fluorescence using FACS analysis. Immunoblotting was performed as described previously [30]. The primary antibodies used for immunoblotting were rabbit polyclonal anti-Cav-1(Santa Cruz Biotechnology Inc, Dallas, Texas), anti-GFP (Santa Cruz Biotechnology Inc), and B-actin protein antibody (Sigma, St. Louis, MO). The secondary antibodies were HRP-linked anti mouse or rabbit (Cell Signaling Technology Danvers, MA). To assay for HIV inhibition lug of plasmid constructs containing Cav-1 were co-transfected along with 1 ug of NL4-3 provirus DNA plasmid (pNL4-3) into 293T cells. Virus production was monitored by reverse transcriptase activity assay in triplicate as described previously using 10ul supernatant of cell culture samples [33]. Cell viability was monitored using Cell Signaling Technology's XTT (2,3-bis(2-methoxy-4-nitro-5-sulfophenyl)-2 H-tetrazolium-5-carboxanilide) Cell Viability Kit (Danvers MA) according to manufactures described protocol.

\section{Foamy Virus Vector Mediated Cav-1 Transduction and HIV Challenges}

To prepare foamy virus vector for Cav-1 transduction into HIV target cells 20 ug of each of the foamy vectors were co-transfected with the packaging constructs pCIgag (20 ug), pCIpol (4 ug), and pClenv ( $2 \mathrm{ug}$ ) into 293T cells per T-75 culture flask as described previously [32]. Transfected cells were maintained in culture for 4 days and supernatants harvested by centrifugation at $5000 \mathrm{rpm}$ for 20 minutes and then by filtering through a 0.45 um filter. Vector particles were concentrated by 100 -fold using $150 \mathrm{kDa}$ Apollo Centrifugal Spin Concentrators (Orbital Biosciences, Topsfield, MA). Concentrated virus particles were tittered by infecting fresh $293 \mathrm{~T}$ cells seeded at a density of $2.5 \times 10^{4}$ per well in 24 wells and counting GFP positive cells. NL4-3 HIV target cells U87-CD4-CXCR4 were seeded at a density of $5 \times 10^{5}$ per well and infected with each of packaged foamy virus vectors at multiplicity of infection (moi) of 10 by spin inoculation twice as described previously [34]. The HIV NL4-3 strain was tittered using the TZM-bl indicator cells and counting Tat induced $\beta$-Gal gene expression before challenge. Foamy virus transduced U87-CD4-CXCR4 cells were challenged 48 hours later with HIV NL4-3 at an moi of 0.005 using the DEAE-dextran method as reported previously [33]. The level of HIV production was monitored from foamy virus vector transduced cells using reverse transcriptase assay.

\section{RESULTS}

\section{Inhibition of HIV Replication by Cav-1 Expressed Under the Control of the HIV LTR}

The cav-1 coding sequence was placed under the control of the HIV LTR (LTR-Cav-1), Fig. (1) for regulated Cav-1 expression in HIV infected cells to determine its ability to inhibit HIV replication. For comparison cav-1 was also placed under a CMV promoter for constitutive expression (CMV-Cav-1). 293T cells were transfected with LTR-Cav-1 or CMV-Cav-1 along with NL4-3 provirus DNA (pNL4-3) and the replication of HIV was monitored for 4 days by harvesting supernatants and assaying for reverse transcriptase. Controls lacking the coding sequence of cav-1 (LTR-IR or CMV-IR) were also co-transfected with pNL4-3 and the level of HIV replication was determined in the same manner. Virus replication was compared in Cav-1 expressing cells transfected with LTR-Cav-1 or CMV-Cav-1 with that of cells transfected with corresponding control vectors LTRIR or CMV-IR, respectively. As shown in Fig. (2A), inhibition of HIV by Cav-1 under the control of the CMV promoter reached $86 \%$ compared to the control CMV-IR. Similarly Cav-1 expression by the HIV LTR was able to reduce virus replication by $90 \%$ as compared with the corresponding control. These results suggest that regulated expression of Cav-1 in HIV infected cells that effectively inhibits virus replication can be achieved with the level of Cav-1 expression being comparable to that of the expression of Cav-1 directed by a constitutive CMV promoter. Such a level of reduction of HIV replication in lieu of Cav-1 suppresses LTR mediated expression indicates that it is an effective inhibitor of virus replication.

\section{Reducing LTR Driven Basal Expression of Cav-1 in the Absence of Tat}

The level of LTR driven background expression of Cav-1 in uninfected cells is critical whether Cav-1 can be used for HIV therapeutics since alterations in Cav-1 expression can be pathogenic $[14,21]$. As show in Fig. (2B) Cav-1 expression is present in cells where LTR-Cav-1 was transfected in the absence of pNL4-3 and such levels of Cav1 expression could be toxic to uninfected cells. Since the cells we used to test our constructs do not express Cav-1, as demonstrated by the control construct, the amount of Cav-1 seen by immunoblot with LTR-Cav-1 in the absence of pNL4-3 represents the level of expression above endogenous levels of cells expressing Cav-1. To overcome this problem we constructed a series of cav-1 expression cassettes and examined the level of Cav-1 in the presence and absence of Tat. First, we placed GFP upstream of Cav-1 while inserting an IRES in between GFP and Cav-1 for LTR regulated expression (LTR-GFP-IR-Cav-1, Fig. (1A). This construct was co-transfected with or without pTatz, a Tat expressing plasmid, and the level of Cav-1 and GFP expression was determined by immunoblots. As shown in Fig. (2C), a significant level of Cav-1 expression was observed in the absence of Tat. A second IRES sequence was included 
A.

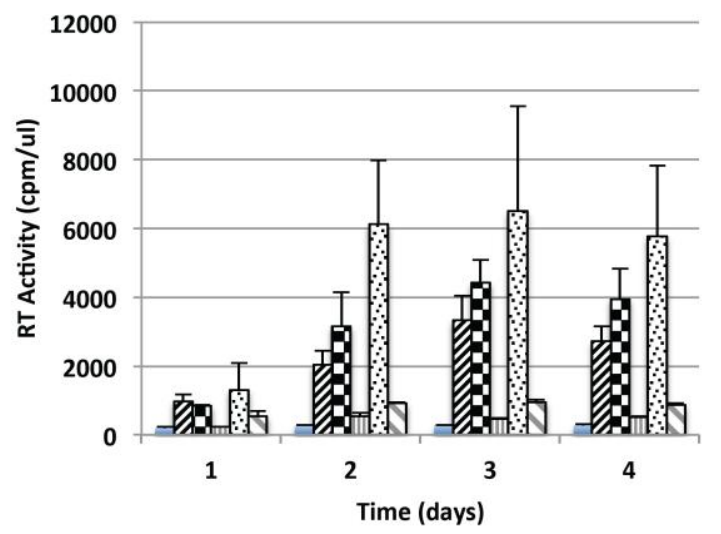

Mock

Z NL4-3

口 LTR-IR

口LTR-Cav-1

ĐCMV-IR

口CMV-Cav-1

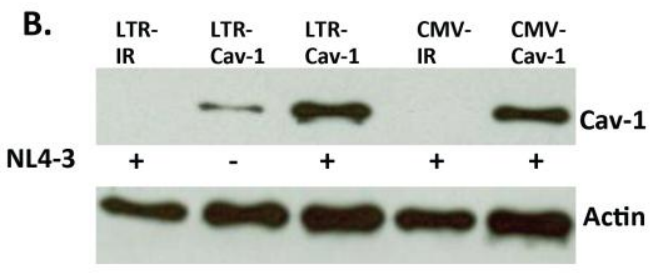

C.
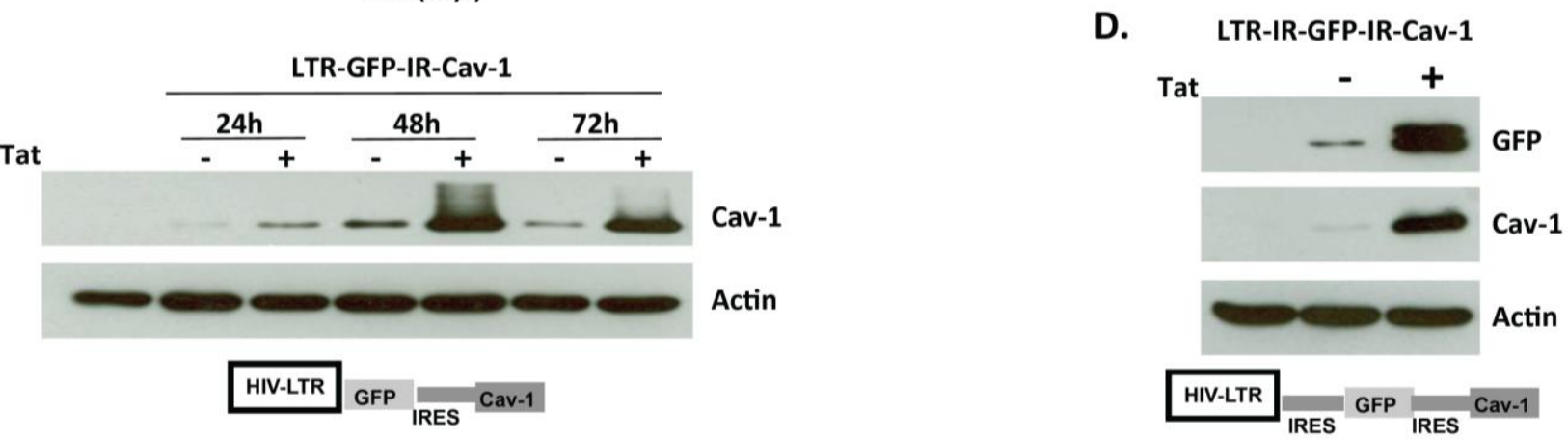

Fig. (2). HIV LTR driven Cav-1 expression and inhibition of HIV replication. Cav-1 was placed under the control of CMV or the HIV LTR promoter for expression and each was co-transfected with the NL4-3 provirus DNA into 293T cells. HIV replication was monitored by reverse transcriptase activity (A) and the expression of Cav-1 by Western blot analysis (B). A series of Cav-1 and GFP expression cassettes were constructed as depicted in the Figure by placing one IRES (LTR-GFP-IR-Cav1) (C) or two IRES (LTR-IR-GFP-IR-Cav-1) (D). Each of these constructs were co-transfected into 293T cells with or without the Tat expression plasmid, pTatz. The expression levels of Cav-1 and GFP were examined by Western blot analysis 48 hours post transfection. Results are expressed as the means \pm standard deviations (SD) from three determinations $(P<0.05)$.

upstream GFP (Fig. (1A), LTR-IR-GFP-IR-Cav-1). The LTR-IR-GFP-IR-Cav-1 construct revealed a significant reduction in Cav-1 expression in the absence of Tat compared to LTR-GFP-IR-Cav-1 while maintaining a high level of expression in the presence of Tat (Fig. 2D). Although placing an additional IRES upstream GFP significantly reduced background expression, there is still some Cav-1 present in LTR-IR-GFP-IR-Cav-1 transfected cells in the absence of Tat. We hypothesized extending the gap between GFP and the second IRES with DNA that will produce RNA with secondary structure would the reby reduce leak through expression of Cav-1. To test our hypothesis first we generated a construct where the rev response element (RRE) from NL4-3 HIV is placed upstream of the IRES-GFP sequence LTR-RRE-IR-GFP (Fig. 1B) and monitored GFP expression by FACs analysis and compared with, the same construct but lacking the RRE (LTR-IR, Fig. 1A) in the presence or absence of Tat. As shown in Fig. (3) fluorescent intensity was significantly reduced in the absence of Tat in cells transfected with LTRRRE-IR-GFP compared to that of LTR-IR. Based on this information the RRE sequence was placed between IRESGFP and the second IRES upstream Cav-1 as shown in Fig. (1B), (LTR-IR-GFP-RRE-IR-Cav-1) to further help reduce leak through expression. This construct was tested in the presence or absence of Tat for Cav-1 expression. As shown in Fig. (4A) no Cav-1 expression was observed in the absence of Tat as determined by Western blot analysis while maintaining the ability of high level Cav-1 expression in cells receiving pTatz. The LTR-IR-GFP-RRE-IR-Cav-1 construct was tested for its' ability to inhibit HIV replication by co-transfecting with pNL4-3 into $293 \mathrm{~T}$ cells (Fig. 4B). Inhibition of HIV replication reached up to $90 \%$ in cells transfected with LTR-IR-GFP-RRE-IR-Cav-1 which is comparable to that of CMV-Cav-1. Cells transfected with LTR-IR-GFP-RRE-IR-Cav-1 remained healthy similar to mock transfected cells in the absence of HIV infection establishing there is no toxicity associated when Cav-1 is placed under a restrictive expression system (Fig. 4C). These results suggest that using a LTR-IR-GFP-RRE-IR-Cav-1 construct, undetectable levels of LTR driven Cav-1 expression can be established in the absence of Tat and HIV infection as determined by Western blots which allows efficient expression of Cav-1 mediated by Tat subsequently effectively inhibiting HIV replication.

\section{Inhibition of Virus Replication by Inducible Cav-1 Expression in HIV Target Cells}

The LTR-IR-GFP-RRE-IR-Cav-1 cassette was placed into foamy virus vector (SFV/LTR-IR-GFP-RRE-IR-Cav-1) to determine its' ability to inhibit virus replication in HIV target cells. A recombinant foamy vector containing Cav-1 


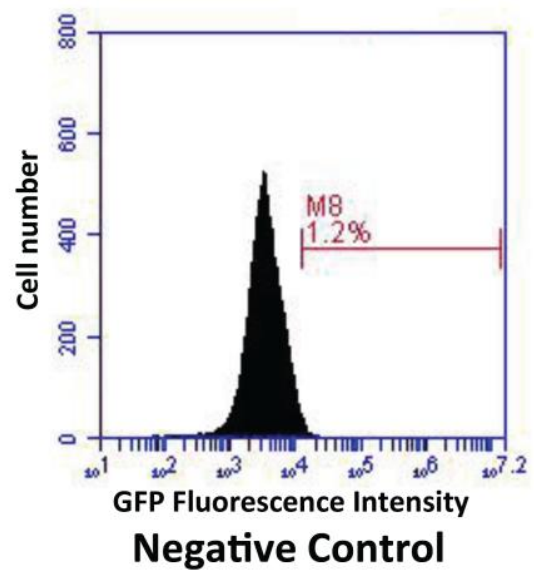

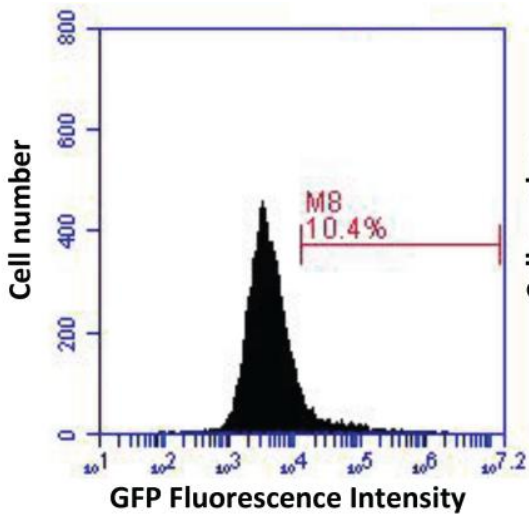

(-) Tat

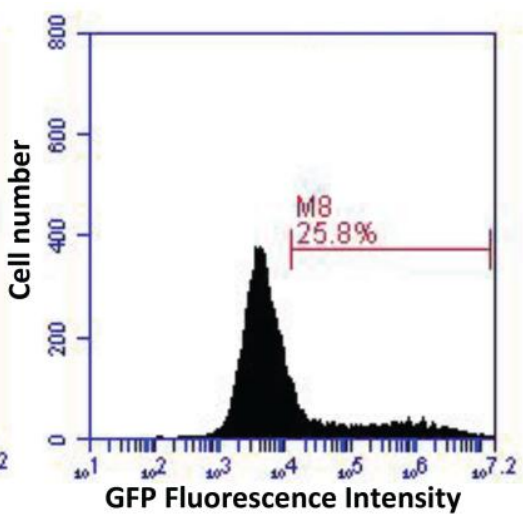

(+) Tat

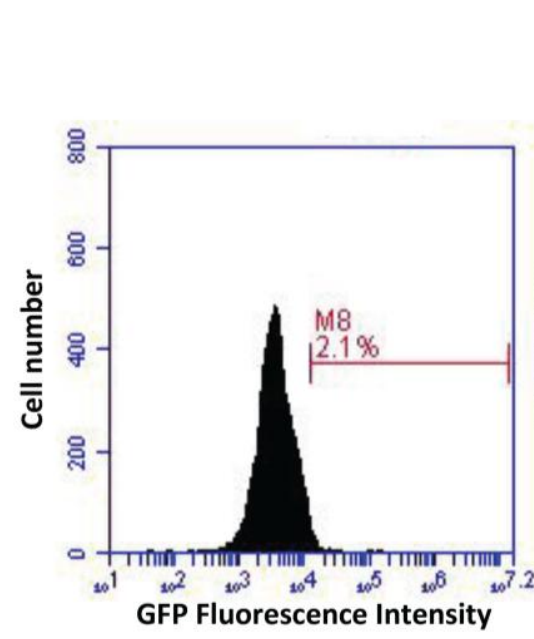

(-) Tat

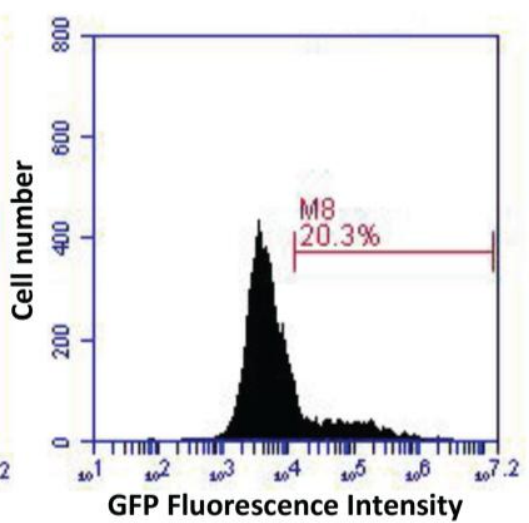

(+) Tat

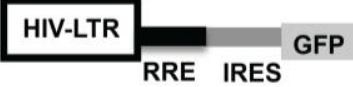

LTR-RRE-IRES-GFP

Fig. (3). Reduction of LTR driven GFP basal expression in in the absence of Tat. The IRES sequence alone or in combination with the HIV Rev response element (RRE) was placed between the HIV LTR and GFP. Each of these constructs were transfected into $293 \mathrm{~T}$ cells and the level of GFP expression was examined by FACS measuring mean fluorescent intensity.

with CMV promoter was also constructed as a control SFV/CMV-Cav-1. An additional control of a foamy virus vector containing a microRNA based siRNA targeting rev (SFV/HL-R) that effectively inhibits HIV replication and a negative foamy virus control expressing GFP (pEGFPD) were included in our studies. Also included, as a control, was HIV infected cells with no foamy virus vector transduction [mock (NL4-3 only)]. Each of these constructs were transfected with the packaging plasmids pCIgag, pCIpol, and pCIenv to generate vector particles and infect the HIV target U87-CD4-CXCR4 cells. The level of HIV replication was analyzed at intervals for 17 days by harvesting supernatants and assaying for reverse transcriptase activity. As shown in Fig. (5), in the mock transduced cells HIV replication was evident at day 3 and rising significantly at day 5 then plateauing at days 7,9 and 11 until cell lysis began. HIV replication in the pEGFPD transduced control had a slow start, peaked at day 9 and 11. In Cav-1 transduced cells whether Cav-1 expression was under the CMV or the HIV LTR promoter the levels of HIV replication were significantly reduced as compared to that of the controls that were mock or pEGFPD transduced (days 5-11). The level of inhibition of HIV replication reached $86 \%$ and $82 \%$ in cells transduced with SFV/CMV-Cav-1 and SFV/LTR-IR-GFPRRE-IR-Cav-1, respectively, compared to that of cells receiving $\mathrm{pEGFPD}$ or mock. This level of inhibition is comparable to that of cells transduced with our positive control SFV/HLR foamy virus vector (days 5-11), a reduction in HIV replication reaching 91\%. The Cav-1 expression in HIV infected cells under the control of the HIV LTR is, therefore, an effective inhibitor of HIV replication.

\section{DISCUSSION}

The use of cellular proteins against HIV are an upcoming and evolving genetic approach that can be included in gene therapy combinations to control HIV infection to the point of a cure. Anti-HIV cellular proteins are important mainly for the reason that their targets are not viral genome that constantly evolve and therefore can safeguard against escape mutants. Cav-1 is one such protein which effectively inhibits HIV replication. Regulated expression of Cav-1 is important 
A. LTR-IR-GFP-RRE-IR-Cav-1

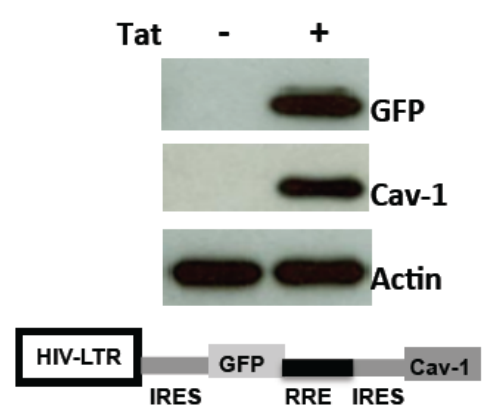

B.

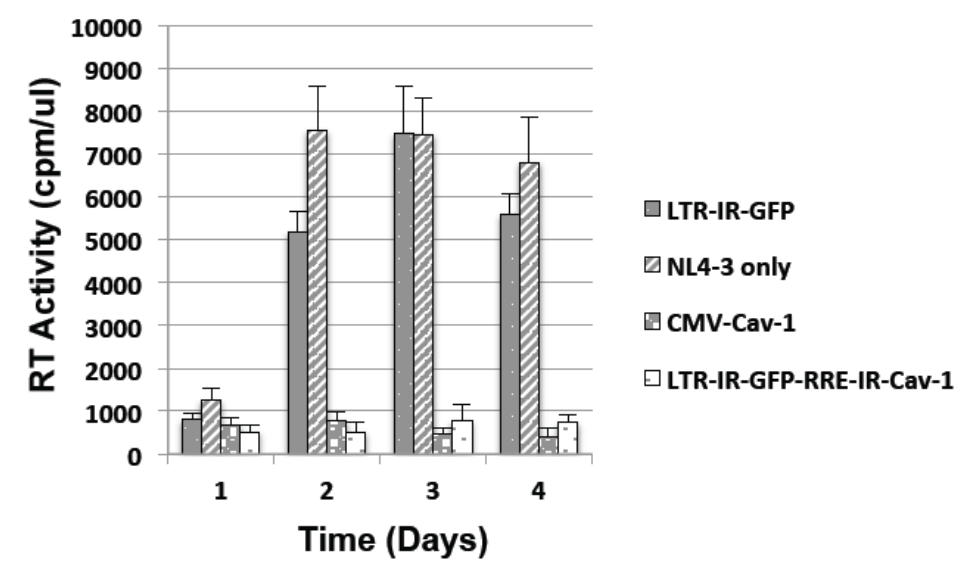

C.

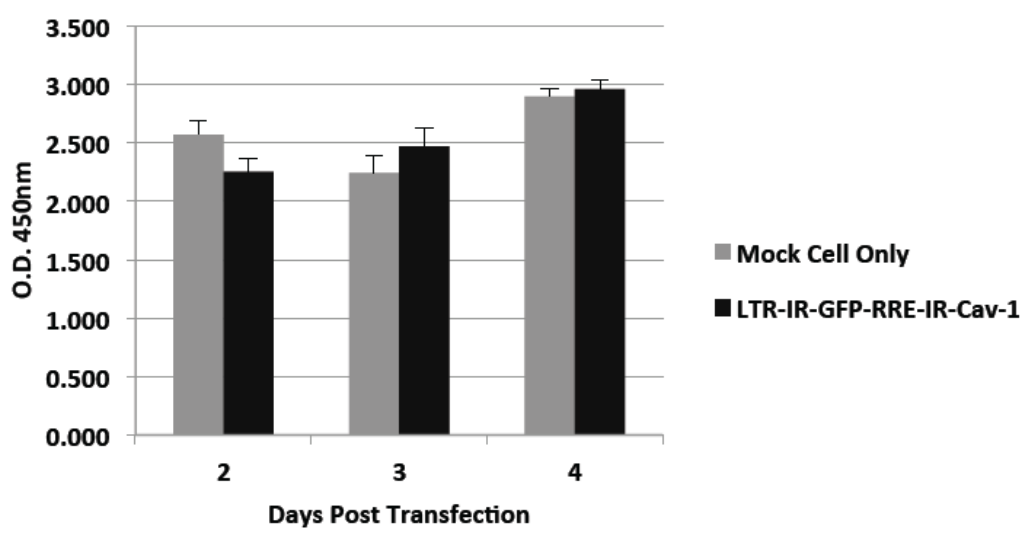

Fig. (4). Inducible expression of Cav-1 with significant reduction in basal level leakage expression. A Cav-1 and GFP expression cassette was constructed as depicted in the Figure by using 2 IRES and an RRE (LTR-IR-GFP-RRE-IR-Cav-1) sequences (A). This construct was cotransfected into 293T cells with or without Tat expression plasmid, pTatz. The expression levels of Cav-1 and GFP were examined by Western blot analysis. (B) Cav-1 expression from LTR-IR-GFP-RRE-IR-Cav-1 was examined for efficacy of inhibition of HIV replication by co-transfecting with NL4-3 and monitoring HIV replication by reverse transcriptase assay. (C) The XTT cell viability assay (Cell Signaling Technology, Danvers MA) was used to determine the nontoxicity and safety of LTR-IR-GFP-RRE-IR-Cav-1. Results are expressed as the means \pm standard deviations (SD) from three determinations $(P<0.05)$.

for safe and effective use of Cav-1 for HIV therapeutics. We have placed the cav-1 gene under the HIV LTR promoter and determined that it efficiently inhibits HIV replication. The expression of Cav-1 in these studies were Tat dependent and the effective inhibition of virus replication suggests that any negative regulation on the HIV LTR driven expression of the antiviral genes is not sufficient enough to have significant impact on the role of Cav-1 in the inhibition of HIV replication. Similar levels of inhibition of HIV replication were observed when cav-1 was placed under the control of a CMV promoter. Our results show no LTR driven Cav-1 expression by Western blot analysis in the absence of Tat or HIV infection revealing a significant reduction of leak through expression in the absence of Tat. This was accomplished by inserting an IRES sequence upstream the coding sequences of each of GFP and Cav- 1 and adding a Rev response element between GFP and the IRES placed at the 5 'end of cav-1.

The absence of basal Cav-1 expression as determined by Western blot analysis with LTR-IR-GFP-RRE-IR-Cav-1 as compared to LTR-GFP-IR-Cav-1 and LTR-IR-GFP-IR-Cav1 could be due to distance spacing with RRE, the RRE structure, and/or weaker ribosomal entry for Cav-1 expression as compared to that of the one that is placed upstream the GFP. Since the amount of leak through of long transcripts is significantly low in the absence of Tat the expression of Cav-1 protein can be extremely low with the additional IRES sequence and the RRE. Cav-1 expression could be below the detection level of Western blots in the absence of Tat. If that is the case, this level of expression is therefore significantly lower than endogenous levels in cells expressing Cav-1 which can easily be seen by Western blots [28-30, 35]. The observation that Cav-1 was not detected by Western blot analysis with our constructs whereas endogenous expression of Cav-1 is easily observed in the absence of Tat or HIV infection indicates that the strategy applied for Cav-1 expression could significantly reduce any safety concerns of toxicity due to transgene expression in uninfected cells. Furthermore, such a strategy will have broad applications and can be beneficial for other hosttargeted interventions as therapeutics.

As described above one of the mechanisms of Cav-1's ability of inhibiting HIV replication is through suppressing gene expression by modulating the NF- $\mathrm{kB}$ pathway and the 


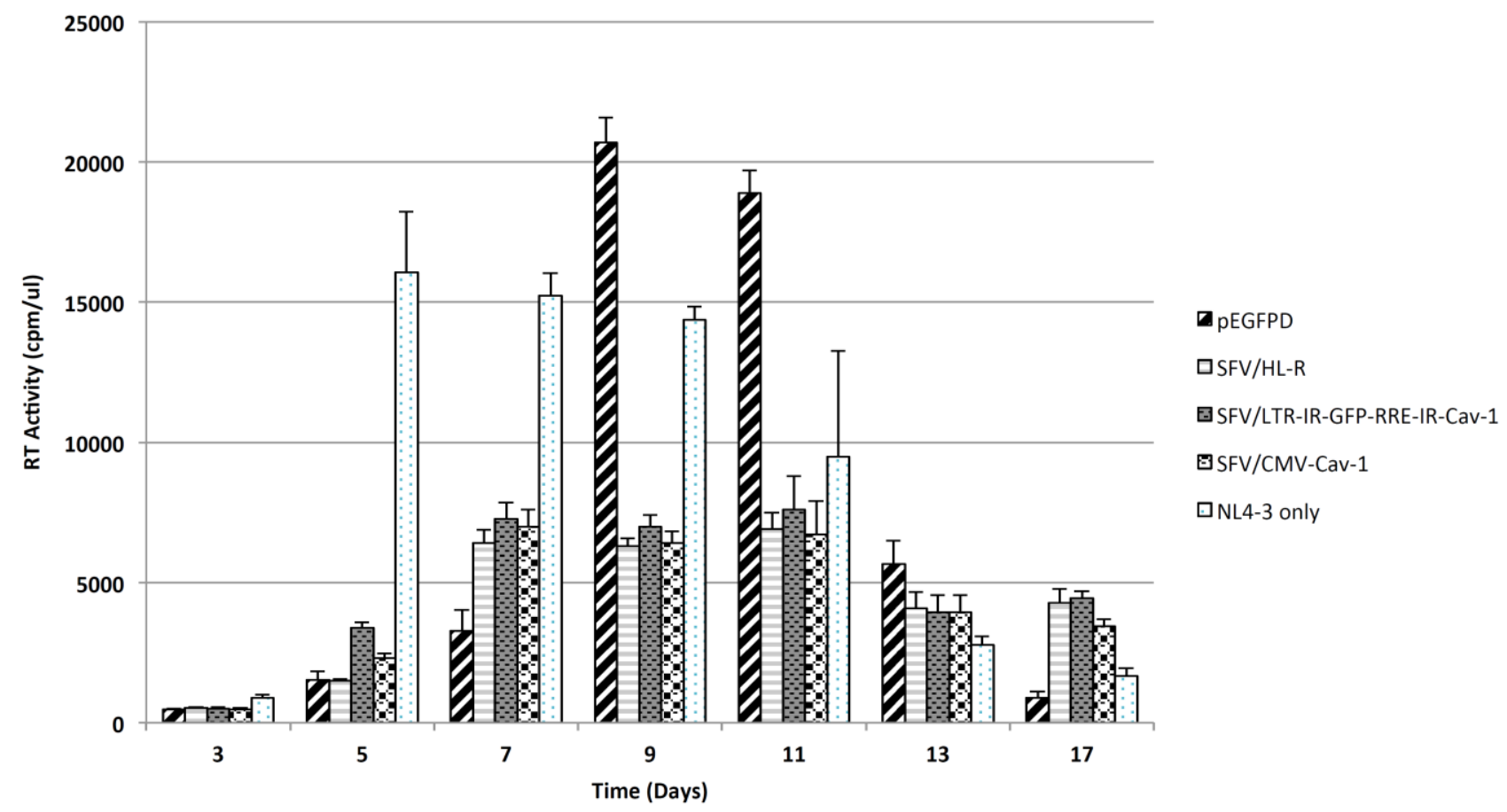

Fig. (5). Inhibition of virus replication in HIV target cells by conditionally expressed Cav-1. The LTR-IR-GFP-RRE-IR-Cav-1 construct was placed in the foamy virus vector for transduction of HIV target cells to determine inhibition of virus replication by conditionally expressing Cav-1 in infected cells. Foamy virus vector mediated transductions of positive (SFV/HL-R and SFV/CMV-Cav-1) and negative (pEGFPD and pNL4-3) controls are included for comparisons. Results are expressed as the means \pm standard deviations (SD) from three determinations $(P<0.05)$.

two NF- $\mathrm{KB}$ sites in the $\mathrm{U} 3$ region of the LTR are critical for Cav-1 mediated inhibition of viral gene expression [29]. Interestingly, although Cav-1 can suppress its own expression through this mechanism our results show that the level of LTR driven expression remains effective enough to inhibit HIV replication. This result suggests the major inhibition of HIV replication is perhaps mediated through the other two mechanisms of Cav-1's ability of promoting cholesterol efflux and blocking of envelope fusion to target cells subsequently arresting the replication cycle. The inhibition mediated by the NF- $\kappa \mathrm{B}$ pathway, therefore, is not sufficient enough to significantly impact its ability to inhibit HIV replication. Alternatively, there may always be adequate levels of Cav-1 expression that results in effective inhibition of viral expression even under a possibility of feed back suppression of its own expression. In support of this notion, LTR driven siRNA expression targeting the $\mathrm{R}$ region of the LTR is shown to effectively inhibit HIV replication [32]. Furthermore, several studies show RNA based anti-HIV approaches such as ribozymes, shRNAs, and antisense RNA expressed from the HIV LTR to effectively inhibit viral replication in HIV infected cells [36-39].

\section{CONCLUSION}

Innate immune antiviral factors have important implications in HIV therapeutics and there is a continual interest in understanding and transferring these molecules into preclinical therapeutic interventions. To this endeavor steps for preclinical studies have already been the case for HIV restriction factors APOBEC $3 \mathrm{G}$, TRIM $\alpha$ and tetherin [40]. The expressions of innate immune antiviral vectors do get activated during viral protection for the first line of defense. Cav-1 expression is also augmented by HIV infection in macrophages and microglial HIV targets and similar to innate antiviral factors Cav-1 suppresses HIV replication. In fact, such bidirectional association of Cav-1 with HIV can contribute to macrophage persistent infection [28-30, 35]. Therefore, like the HIV restriction factors and other antiviral innate immune molecules Cav-1 can be important for clinical therapeutic intervention. The results presented here using a cellular protein, $\mathrm{Cav}-1$, as a gene therapy target in a cell culture system will be a basis to test its therapeutic potential in preclinical settings using animal models.

\section{CONFLICT OF INTEREST}

The authors confirm that this article content has no conflict of interest.

\section{ACKNOWLEDGMENTS}

This research was supported by a grant from the National Institutes of Health (AI39126) to A.M.

\section{REFERENCES}

[1] Kurzchalia TV, Dupree P, Parton RG, et al. VIP21, a 21-kD membrane protein is an integral component of trans-Golginetwork-derived transport vesicles. J Cell Biol 1992; 118: 1003-14.

[2] Dupree P, Parton RG, Raposo G, Kurzchalia TV, Simons K. Caveolae and sorting in the trans-Golgi network of epithelial cells. EMBO J 1993;12:1597-605.

[3] Palade GEFsobc. Fine structure of blood capillaries. J Appl Phys 1953; 24:1424. 
[4] Yamada E. The fine structures of the gall bladder epithelium of the mouse. J Biophys Biochem Cytol 1955; 1: 445-58.

[5] Stan RV. Structure of caveolae. Biochem Biophys 2005; 1746: 334-48.

[6] Stan RV, Tkachenko E, Niesman IR. PV1 is a key structural component for the formation of the stomatal and fenestral diaphragms. Mol Biol Cell 2004; 15: 3615-30.

[7] Parton RG, Simons K. The multiple faces of caveolae. Nat Rev Mol Cell Biol 2007; 8: 185-94.

[8] Lisanti MP, Tang Z, Scherer PE, Kübler E, Koleske AJ, Sargiacomo M. Caveolae, transmembrane signalling and cellular transformation. Mol Membr Biol 1995; 12: 121-4.

[9] Okamoto T, Schlegel A, Scherer PE, Lisanti MP. Caveolins, a family of scaffolding proteins for organizing "preassembled signaling complexes" at the plasma membrane. J Biol Chem 1998; 273: 5419-22.

[10] Kurzchalia TV, Parton RG. Membrane microdomains and caveolae. Curr Opin Cell Biol 1999; 11: 424-31.

[11] Liu P, Rudick M, Anderson RG. Multiple functions of caveolin-1. J Biol Chem 2002; 277: 41295-8.

[12] Shin JS, Z. Gao, Abraham SN. Involvement of cellular caveolae in bacterial entry into mast cells. Science 2000; 289: 785-88.

[13] Pelkmans L, Kartenbeck J, Helenius A . Caveolar endocytosis of simian virus 40 reveals a new two-step vesicular-transport pathway to the ER. Nature Cell Biol 2001, 3: 473-83.

[14] Williams TM, Lisanti MP. Caveolin-1 in oncogenic transformation, cancer, and metastasis. Am. J. Physiol. Cell Physiol 2005; 288: C494-506

[15] Frank PG, Pavlides S, Cheung MW, Daumer K, Lisanti MP. Role of caveolin-1 in the regulation of lipoprotein metabolism. Am J Physiol Cell Physiol 2008; 295: C242-8.

[16] Frank PG, Pavlides S, Lisanti MP. Caveolae and transcytosis in endothelial cells: role in atherosclerosis. Cell Tissue Res 2009; 335: 41-7.

[17] Gargalovic P, Dory L. Caveolins and macrophage lipid metabolism. Journal of Lipid Research 2002; 44: 11-21.

[18] Harris J, Werling D, Hope JC, Taylor G, Howard CJ. Caveolae and caveolin in immune cells: distribution and functions. Trends Immunol 2002; 23: 158-64.

[19] Harris J, Werling D, Koss M, Monaghan P, Taylor G, Howard CJ. Expression of caveolin by bovine lymphocytes and antigenpresenting cells. Immunology 2002; 105: 190-5.

[20] Quest AFG, Leyton L, Párraga M. Caveolins, caveolae, and lipid rafts in cellular transport, signaling, and disease. Biochem. Cell Biol 2004; 82: 129-44.

[21] Mercier I, Jasmin J-F, Pavlides S, et al. Clinical and translational implications of the caveolin gene family: lessons from mouse models and human genetic disorders. Laboratory Investigation 2009; 89: 614-23.

[22] Couet J, Li S, Okamoto T, Ikezu T, Lisanti MP. Identification of peptide and protein ligands for the caveolin-scaffolding domain. Implications for the interaction of caveolin with caveolaeassociated proteins. J Biol Chem 1997; 272: 6525-33.

[23] Hovanessian AG, Briand JP, Said AS, et al. The caveolin-1 binding domain of HIV-1 glycoprotein gp41 is an efficient B-cell epitope vaccine candidate against virus infection. Immunity 2004; 21 : 61727.

[24] Huang JH, Lu L, Hong L, Chen X, Jiang S, Chen Y-H. Identification of the HIV-1 gp41 Core-binding Motif in the
Scaffolding Domain of Caveolin-1. J Biol Chem 2007, 282: 614352.

[25] Wild C, Dubay JW, Greenwell T, et al. Propensity for a leucine zipper-like domain of human immunodeficiency virus type $1 \mathrm{gp} 41$ to form oligomers correlates with a role in virus-induced fusion rather than assembly of the glycoprotein complex. Proc Natl Acad Sci USA 1994; 91: 12676-80.

[26] Melikyan GB, Markosyan RM, Hemmati H, Delmedico MK, Lambert DM, Cohen FS. Evidence that the transition of HIV-1 gp41 into a six-helix bundle, not the bundle configuration, induces membrane fusion. J Cell Biol 2000; 151: 413-23.

[27] Llano M, Kelly T, Vanegas M, et al. Blockade of Human Immunodeficiency Virus Type 1 Expression by Caveolin-1. J Virol 2002; 76: 9152-64.

[28] Wang XM, Nadeau PE, Lo Y-T, Mergia A. Caveolin-1 modulates HIV-1 envelope induced bystander apoptosis through gp41. J Virol 2010; 84: 6515-26.

[29] Wang X-M, Nadeau PE, Lin S, Abbott JE, Mergia A. Caveolin 1 inhibits HIV replication by transcriptional repression mediated through NF-\{kappa\}B. J Virol 2011; 85: 5483-93.

[30] Lin S, Nadeau PE, Wang X, Mergia A. Caveolin-1 reduces HIV-1 infectivity by restoration of HIV Nef mediated impairment of cholesterol efflux by apoA-I. Retrovirology 2012; 9: 85.

[31] Simmons GE, Jr., Taylor HE, Hildreth JE. Caveolin-1 suppresses human immunodeficiency virus-1 replication by inhibiting acetylation of NF-kappaB. Virology 2012; 432: 110-9.

[32] Park J, Nadeau PE, Mergia A. Activity of TAR in inducible inhibition of HIV replication by foamy virus vector expressing siRNAs under the control of HIV LTR. Virus Res 2009; 140: 11220.

[33] Park J, Nadeau P, Zucali JR, Johnson CM, Mergia A. Inhibition of simian immunodeficiency virus by foamy virus vectors expressing siRNAs. Virology 2005; 343: 275-82.

[34] Mergia A, Soumya C, Kolson DL, Goodenow MM, Ciccarone T. The efficiency of simian foamy virus vector type-1 (SFV-1) in nondividing cells and in human PBLs. Virology 2001; 280: 243-52.

[35] Lin S, X-M W, Nadeau PE, Mergia A. HIV infection upregulates Caveolin 1 (Cav-1) expression to restrict virus production. J Virol 2010; 84: 9487-96.

[36] Dropulic B, Hermankova M, Pitha P. A conditionally replicating HIV-1 vector interferes with wild-type HIV-1 replication and spread. Proc Natl Acad Sci USA 1996; 93: 11103-8.

[37] Paik SY, Banerjea A, Chen CJ, Ye Z, Harmison GG, Schubert M. Defective HIV-1 provirus encoding a multi-target ribozyme inhibits accumulation of spliced and unspliced HIV-1 mRNAs, reduces infectivity of viral progeny, and protects the cells from pathogenesis. Hum Gene Ther 1997; 8: 1115-23.

[38] Unwalla HJ, Li HT, Bahner I, Li MJ, Kohn D, Rossi JJ. Novel pol ii fusion promoter directs human immunodeficiency virus type 1inducible coexpression of a short hairpin rna and protein. J Virol 2006; 80: 1863-73.

[39] Unwalla HJ, Li MJ, Kim JD, et al. Negative feedback inhibition of HIV-1 by TAT-inducible expression of siRNA. Nat Biotechnol 2004; 22: 1573-8.

[40] Sloan RD, Wainberg MA. Harnessing the therapeutic potential of host antiviral restriction factors that target HIV. Expert Rev Anti Infect Ther 2013;11:1-4

This is an open access article licensed under the terms of the Creative Commons Attribution Non-Commercial License (http://creativecommons.org/licenses/by-nc/3.0/) which permits unrestricted, non-commercial use, distribution and reproduction in any medium, provided the work is properly cited. 